\title{
PARAPLEGIA AFTER CORONARY BYPASS OPERATIONS: RELATIONSHIP TO SEVERE HYPERTENSION AND VASCULAR DISEASE
}

Neil J. Thomas, MD, and Adrian T. Harvey, BS, Phoenix, Ariz

Spinal cord infarction is an unusual clinical event in the postoperative period. It has been described after general and orthopedic procedures in otherwise healthy patients in whom hypotension develops for a variety of reasons. ${ }^{1}$ It has also been described in the context of other neurologic complications of cardiac operations and appears to be rare in this setting. ${ }^{2}$ We report paraplegia and spinal cord injury in two patients with severe peripheral vascular disease who had hypertensive crises after otherwise routine coronary revascularization.

From the Cardiovascular Research and Care Foundation, Inc, Phoenix, Ariz.

Received for publication Oct 26, 1998; accepted for publication Dec 2, 1998.

Address for reprints: Neil J. Thomas, MD, Cardiovascular Research and Care Foundation, Inc, 2720 North 20th St, Suite 440, Phoenix, AZ 85006. E-mail: njtcvsg@ @abilnet.com.

J Thorac Cardiovasc Surg 1999;117:834-6

Copyright $\odot 1999$ by Mosby, Inc.

0022-5223/99 $\$ 8.00+0 \quad \mathbf{1 2 / 5 4 / 9 6 2 1 1}$

\section{Clinical summaries}

PATIENT 1. A 65-year-old woman with known coronary artery disease, hypertension, diabetes mellitus, and severe, symptomatic aorto-iliac occlusive disease was admitted to the hospital with an acute inferior wall myocardial infarction. An aortic balloon could not be inserted. Catheterization of the left side of the heart showed significant 3-vessel disease with normal left ventricular function. Laboratory work-up revealed evidence of mild chronic renal insufficiency (baseline creatinine value: $1.8 \mathrm{mg} / \mathrm{dL}$ ) and mild anemia (hemoglobin value: $10 \mathrm{mg} / \mathrm{dL}$ ). Several days later, she underwent routine 3-vessel coronary bypass grafting using the left internal thoracic artery to bypass the left anterior descending coronary artery. She was weaned from bypass without difficulty. However, for approximately 30 minutes, her mean blood pressure was above $100 \mathrm{~mm} \mathrm{Hg}$ and extremely difficult to control before decannulation, even with intravenous nitroprusside. Postoperative recovery was otherwise satisfactory; however, she awoke from anesthesia with a spinal cord injury at the sixth thoracic segmental level and paraplegia. Com- 
puted tomographic scan of the chest revealed no evidence of aortic dissection but showed a highly calcified thoracic and abdominal aorta.

PATIENT 2. A 59-year-old man with a history of intermittent claudication, bilateral superficial femoral artery occlusions, and lupus erythematosus, inactive for 15 years, was admitted with the diagnosis of unstable angina. On presentation, an aortic balloon was inserted, intravenous heparin infusion was initiated, and he was referred for surgery urgently after significant 3-vessel coronary disease was observed in the setting of accelerating anginal symptoms. He was a heavy smoker. He had a history of stable angina and severe hypertension for which he received several medications. Laboratory values were within normal limits. Four-vessel bypass using the left internal thoracic artery for the left anterior descending coronary artery proceeded uneventfully. On admission to the intensive care unit, the mean blood pressure was greater than $100 \mathrm{~mm} \mathrm{Hg}$. Initial postoperative cardiac index was marginal $(2.0 \mathrm{~L} / \mathrm{min}$ per square meter) and elevated systemic resistance prompted the administration of a loading dose of intravenous milrinone. This readily controlled the elevated pressure, temporarily, and improved the cardiac index. The balloon pump was discontinued immediately because of the development of ischemia in his left foot. This was consistent with the preoperative clinical impression of significant femoral and perhaps iliac level atheroma. The lower extremity improved, but hypertension recurred. Mean blood pressure was recorded at between 100 and $110 \mathrm{~mm} \mathrm{Hg}$ during the subsequent 6-hour period. Intravenous nitroglycerin was used for control and was variably effective. He was successfully extubated 12 hours after the operation, and his oral antihypertensive medications were reinstituted with good effect. Paraplegia was noted for the first time 2 hours later during an attempt to move the patent to a chair. Neurology consultation was obtained, and his level of injury was identified at the ninth thoracic vertebra. Computed tomographic scan of the aorta showed nearly circumferential, but irregular, calcification and no evidence of dissection. Myelography, looking for a compressive lesion of the cord, showed no abnormalities. He had an otherwise uneventful postoperative course and was discharged on day 10 .

Discussion. Spinal cord injury is rare after coronary bypass operations, but reports of paraplegia and quadriplegia have been published in the literature. ${ }^{2,3}$ The cases presented here suggest an association between profound and difficult-tocontrol hypertension, severe preexistent peripheral vascular disease, and the postoperative development of spinal cord infarction. We are aware of no such previous description of this association. However, other authors have alluded to comparisons with other forms of thoracic aortic catastrophe in which paraplegia is known to occur. Archer and associates ${ }^{4}$ described the effects of acute aortic dissection after cardiac surgery but recognized the rarity of spinal cord ischemia, even in the aftermath of this event.

Since the earliest days of the use of cardiopulmonary bypass for the performance of cardiac surgery, overt postoperative neurologic deficit has been one of the most dreaded and devastating complications. When Javid and colleagues ${ }^{5}$ reviewed their experience regarding such events, significant hypotension during cardiopulmonary bypass, the harmful effects of protracted extracorporeal circulation, and the contribution of microemboli were considered contributing factors. Their report remains a relatively accurate summary of potential mechanisms nearly 30 years later, but it does not specifically address the issue of spinal cord infarction after coronary grafting. Clearly, hypotension resulting in hypoperfusion, microembolization, and microvascular thrombosis, including that related to severe forms of heparin-induced thrombocytopenia, have been shown, over the years, to be etiologic factors in various forms of stroke syndromes, including spinal cord stroke. ${ }^{3}$ Despite this relative clarity regarding potential mechanisms for the development of neurologic events after cardiac operations, in our experience and from our review of the literature, it appears to be the exception for the cause of a stroke to be clearly elucidated in any given patient.

In these patients, spinal cord infarction appears to have been related to postoperative hypertensive crises associated with atheromatous involvement of the thoracic aorta. The presence of clearly definable, heavily calcified atheroma on computed tomographic evaluation, clinically relevant peripheral vascular disease in both patients, and our review of the pertinent literature all contributed to this impression. Harris and coworkers ${ }^{3}$ reported a case of a 70-year-old man who died of acute spinal cord infarction, which developed after coronary bypass grafting and the intraoperative insertion of an aortic balloon pump. No disruption of the aortic layers was noted at autopsy, but severe ulcerated atheromatous involvement was seen grossly and clear evidence of radicular artery embolization was present at the microscopic level. In the present report, both patients survived and otherwise did well. Consequently, the rupture of an atheromatous plaque or the raising of a localized intimal flap leading to microscopic embolization of debris can only be surmised. Without overt dissection of the thoracic aorta, conventional computed tomographic evaluation cannot fully define the details of such severe involvement of the aorta, as was described at autopsy by Harris and associates. ${ }^{3}$

Despite these limitations, the constellation of clinical and pathologic findings presented here suggest to us that when spinal cord infarction occurs after cardiac surgery, it is related to hypertension rather than hypotension and resembles other thoracic aortic catastrophes in that regard. Aortic plaque rupture related to the development of a hypertensive crisis, with microembolization, appears to be the likely pathogenesis. Furthermore, involvement of the aorta by a localized dissection or plaque rupture does not, in our opinion, require the involvement of the artery of Adamkiewicz, as suggested in the literature. ${ }^{4}$ Either disruption of a plaque anywhere in the thoracic aorta, with distal embolization, or embarrassment of major collateral flow from the aorta or its branches is a sufficient explanation. Although this syndrome remains extremely unusual in the clinical setting, these patients are instructive because of their similarities and because of the apparent cause of their stroke syndromes. The avoidance of significant hypertension, or hypotension, has always been warranted in the perioperative period for 
patients undergoing cardiovascular or other surgical procedures. However, this report suggests that severe hypertension should be vigilantly avoided in patients undergoing cardiopulmonary bypass, particularly when severe peripheral vascular disease is present and significant involvement of the aorta at various levels is unpredictable.

\section{REFERENCES}

1. Singh U, Silver JR, Welply NC. Hypotensive infarction of the spinal cord. Paraplegia 1994;32:314-22.
2. Gonzalez-Scarano F, Hurtig HI. Neurologic complications of coronary artery bypass grafting: case control study. Neurology 1981;31:1032-5.

3. Harris RE, Reimer KA, Crain BJ, Becsey DD, Oldham HN. Spinal cord infarction following intraaortic balloon support. Ann Thorac Surg 1986;42:206-7.

4. Archer AG, Choyke PL, Zeman RK, Green CE, Zuckerman M. Aortic dissection following coronary bypass surgery: diagnosis by CT. Cardiovasc Intervent Radiol 1986;9:142-5.

5. Javid H, Tufo HM, Najafi H, Dye WS, Hunter JA, Julian OC. Neurological abnormalities following open-heart surgery. J Thorac Cardiovasc Surg 1969;58:502-7.

\section{Targeted}

The Journal of Thoracic and Cardiovascular Surgery gives you two tables of contents.

The condensed table of contents tells you at a glance what topics and authors are presented each month. The expanded table of contents gives you a brief abstract of each article. You select only those articles of most interest to you for further reading. 